\title{
MICELLAR MICROPARTICLES: A NOVEL APPROACH TO TOPICAL DRUG DELIVERY SYSTEM
}

\author{
MONISHA BANSAL ${ }^{*}$, SHAHID JAMIL ${ }^{1}$ \\ aDepartment of Pharmaceutics, University School of Pharmaceutical Sciences, Rayat-Bahra University, Mohali, 140104 \\ Email: monishabansal595@gmail.com
}

Received: 24 May 2018, Revised and Accepted: 23 Jun 2018

\begin{abstract}
Topical drug delivery system is defined as the pharmaceutical dosage form which when applied onto the skin provides protection of skin and prevents serious skin disorders. Topical drug are being used for several years and still have its potential in new pharmaceutical technologies investigated. Skin is the most easily accessible organ of the body which has the potential to facilitate the delivery of several drugs with better efficacy, confining the pharmacological or other effect of the drug to the surface of the skin. Micelles are colloidal particles with a size smaller than $100 \mathrm{~nm}$ that allow a great depth of tissue penetration for targeted drug delivery, but rapidly disintegrate in the body. Microparticles containing micelles have the potential for delivering hydrophobic drug encapsulated in micelles on the target site in the specific part of the body. Micellar microparticles allow the improvement of solubility and dissolution of poorly soluble drugs. Microparticles containing micelles have the potential for delivering micelle-encapsulated hydrophobic drugs in targeted therapy. This article reviews the topical drug delivery system, colloidal drug delivery system and aspects and literature reviewed on micellar microparticles and its advantages in pharmaceuticals. An overview of reviews was conducted to locate published literature between 2000 and 2017.
\end{abstract}

Keywords: Micelles, Microparticles, Colloidal microparticles, Hydrophobic drug, Targeting

(C) 2018 The Authors. Published by Innovare Academic Sciences Pvt Ltd. This is an open access article under the CC BY license (http://creativecommons.org/licenses/by/4.0/] DOI: http://dx.doi.org/10.22159/ijap.2018v10i5.27506

\section{INTRODUCTION}

Topical drug delivery system (TDDS) is defined as the pharmaceutical dosage form applied onto the skin directly for the treatment of skin disease. These are intended to confine the pharmacological effect of the drug onto the surface of the skin [1]. The TDDS includes large variety of pharmaceutical dosage form like liquid preparations, semisolids and spray powders. However, the most widely used semisolid preparation for topical drug delivery includes gels, creams and ointments [2]. Topical formulations are applied to the superficial areas such as the skin, eyes, nose and vagina for the treatment of local diseases [3].

\section{Advantages of topical drug delivery}

Topical administration has numerous advantages and can be summarised as follows:

1. Hepatic first-pass metabolism and the GIT metabolism are avoided

2. Offers an alternative pathway for patients who have oral dosing issues, such as in unconscious patients

3. Cutaneous delivery can provide direct access to the diseased or target site such as for fungal skin disease or eczema

4. Fewer side effects related to systemic toxicity compared to other routes of administration

5. Patient compliance and acceptance

6. Ease and convenience of application

7. Painless and non-invasive technique

8. Improvement in drug bioavailability

9. Better physiological and pharmacological response

10. Minimum systemic toxicity and exposure of drug to noninfectious tissue/sites

11. Ability to deliver drug more selectively

12. Enhanced suitability for self-medication [4-6]

TDDS involve the introduction of a drug to the surface of the body, in a formulation which can be absorbed. When the topical formulation is applied onto the skin, it should interact with the skin environment, which could further influence the rate of release of the compound in order to achieve adequate skin absorption [7]. The TDDS are generally applied for the purpose as antiseptics, antifungal agents, skin emollients and protectants [8]. Skin is the main route of topical drug delivery system [9]. Skin is most accessible organ and is potential to facilitate the delivery of several drugs with better efficacy than rest of any other route of administration [8].

In TDDS, drug reaches to the site of action via diffusion and their absorption takes place on the skin [10]. This route is one of the best options for the cutaneous purpose [11]. These systems are often very easy for patients to use, which makes them appealing. In all cases, the goal of a drug delivery system is to get the right dosage to the right place [6]. Many topical preparations, however, contain therapeutically active ingredients which is dispersed or dissolved in the base. The release rates of topical medications depend solely on the physical and chemical, properties of the carrier and the medication used [12-14].

The topical formulations are known for its local dermatological action and it should deliver the medication across the localised area of the skin. To develop an ideal dosage form one must take into account retention of the dosage form, the flux of drug across the skin and patient acceptability of the formulation [15-17]. As topical formulations contain multiple components with active ingredients it should be compatible and should provide stable chemical environment for all the ingredients [7].

The stratum corneum of the skin is very selective as it permits selectively due to which the drug transport through skin becomes limited. The penetrants that have particular physiochemical properties can pass through the skin adequately under passive conditions $[18,19]$.

A topical dermatological product must be aesthetically pleasing, physically and chemically stable and have an adequate shelf life. Topical drug delivery system allows optimum penetration of the drug into the skin membrane.

A successful topical formulation must comply with the following properties: [20-22]

1. Physically and chemically stable having an adequate shelf life

2. Releases drug from the formulation and delivers it into the skin as required for the target indication 


\section{Cosmetically elegant and acceptable to patients}

4. Contains only excipients that are necessary, FDA-approved or acceptable from a regulatory perspective, and acceptable for the disease state

\section{Easy to apply and compatible with the desired packaging}

6. Scalable manufacturing processes on a commercial level [23-26]

\section{Drug delivery across the skin}

The skin is composed of 3 layers being: epidermis, dermis and subcutaneous [27]. The epidermis is the most superficial layer of the skin and is composed of stratified keratinized squamous epithelium which varies in thickness in different parts of the body [28]. The skin being relatively waterproof protects the delicate structure like blood vessels and venous plexus that get the blood inflow from the skin capillaries underneath the skin [29]. The skin is the directly assessable organ for the diagnosis and treatment of various disorders. The drug absorption from a topical formulation involves three primary mechanisms: transcellular, intercellular, and follicular. Most of the drugs pass through the lipid bilayer to viable layers of the skin or through pilosebaceous route. The skin acts as a two-way barrier for prevention and loss of water and electrolytes. The barrier is present in the outermost layer of the epidermis, the stratum corneum [30-36].

The development of topical drug delivery system requires a descriptive knowledge of the therapeutic agents as well as the excipients used in the formulation. The selection of excipients should be such that it can easily penetrate the skin barrier and the drug is delivered to the desired/targeted site $[37,38]$. In topical formulations, here the micellar microparticles are administered onto the skin through cream as a semisolid dosage form. The Microparticles entrapped micelles have the potential to deliver hydrophobic drug encapsulated within micelles on the targeted site.

\section{Colloidal drug delivery system}

Colloidal drug delivery systems (CDDS) is the delivery system containing particulates of Nanometric range which includes niosomes, liposomes, multiple emulsions, nanospheres and ceramics. CDDS are essentially required for the transportation of loaded drug to the targeted/specific site selectively. Colloids are the intermediate kind of solutions whose properties lie between true solution and suspension [39]. A colloidal system is a two-phase system containing a dispersed phase and a continuous phase. A continuous phase is the one in which the very fine particles are suspended known as a dispersed phase. The colloidal dispersions vary from solutions on the basis of particle size of the dispersed phase and its apparent stability. The dispersed phase in the colloidal system is uniformly dispersed in the continuous medium and hence forming a homogenous solution not visible to naked eyes or even with an ordinary microscope. However, the colloidal solution is a heterogeneous dispersion of two immiscible phases when under ultra-microscope [40].

\section{Carriers used in topical drug delivery system}

Colloidal drug carrier is essentially required to transport the loaded drug successfully. They are the drug vectors that sequester and retain the drug delivery route until it is transported at the targeted site. The targeting of the drug at the specific site not only improve the therapeutic efficacy but also reduce the amount of drug incorporated required for therapeutic response and minimizing unwanted toxic effects. The colloidal carriers deliver the drug only to targeted site thereby minimize the overall drug consumption and related side effects to a greater extent. Micellar solutions, liquid crystal dispersions, nanoparticle dispersions, and vesicles are the drug carrier systems consist of small particles of 10-400 nm diameters and showed a great promise as drug delivery systems. The major goal of developing formulations is to obtain a system with optimized drug loading and release properties, longer shelf-life and lower toxicity. The colloidal carrier should be able to cross the anatomical skin barrier effectively. It should be recognized by the target cells specifically and selectively. The carriers should be non-toxic, non-immunogenic; the bio modules should not be ubiquitous $[37,41]$.

\section{Micellization}

The micelles are the aggregates of surfactant molecule that disperse in a liquid colloidal solution [42]. The micelles formation depends solely on the concentration of the surfactant in the polar or nonpolar solvents. The process of forming micelles is called as Micellization. The surfactant when added at lower concentration monomers disperse at the lower surface or aggregate at the interface until the entire surface gets saturated by the surfactants. On further addition of surfactants, the concentration of monomers in the solution increases and it starts forming micelles. This concentration at which the micelles formation starts is called as CMC (Critical Micelle Concentration). CMC is the concentration of monomers at which micelles formation start insolvent at a particular temperature. Micelles form only when the concentration of surfactant is greater than the CMC [43].

Micelles are lipid molecules present in the spherical form in the aqueous solutions. These are supramolecular, non-static structures formed by surfactant molecules in aqueous solutions [44]. The micelles are formed due to the amphipathic nature of the fatty acids which means they contain both hydrophobic and hydrophilic group [45]. They usually contain a polar or hydrophilic head group onto the surface in contact with water. The hydrophobic or non-polar tail forms the inner part of the micelles away from the water surface [46]. Micelles are thermodynamically stable and easily reproducible, but they can be destroyed by water dilution when the surfactant concentration is below its CMC [47].

\section{Types of micelles}

\section{Monomeric Micelles}

2. Reverse micelles

3. Polymeric micelles

\section{Mechanism of drug transport through skin by colloids}

Skin interaction with the colloids occurs either at the skin surface or in the deeper layer of the skin called stratum corneum. Hofland et al., demonstrated the process of adsorption and fusion of vesicles onto the surface which results in the formation of rough structures on top of the outermost corneocytes $[48,49]$. The treatment of the skin with liquid state liposomes and niosomes results in the changes in the stratum corneum. The application of gel state niosomes resulted in no ultrastructural changes [50]. The components of liquid state liposomes and niosomes can enter the deeper layers of the stratum corneum where they can modify the intercellular lipid lamellae, whereas the components of gel state niosomes remain on the skin surface. The liquid state niosomes and liposomes are effective in increasing the drug transport into and across the skin. This is in accordance with a study that found a correlation between the skin penetration and the fluidity of the vesicle bilayers determined by electron spin resonance [51]. It is concluded from the above studies that liposomes and niosomes can penetrate into stratum corneum [52-54].

Remarkably, it was noticed that these liposomes are very flexible lipid vesicles. An increased structure deformability of the lipid bilayers will directly result in skin interactions. The liquid state being structurally deformable can enter the stratum corneum whereas gel state liposomes and rigid liquid vesicles do not enter the stratum corneum as intact entities. There are two major pathway from which the encapsulated drug release from the micellar core. These pathways involve the dissociation of micelles followed by the drug cleavage from the unimer and the drug cleavage within the micelle followed by diffusion out of the drug delivery system. There are various ways to control the cleavage. For micellar dissociation, three mechanisms exist by which the degradation occurs [55]. For drug cleavage followed by diffusion, the release depends on the chemical conjugation of the drug to the hydrophobic polymer [56].

\section{Cloud point technique (CPT)}

The cloud point technique (CPT) is a phenomenon in which an aqueous solution of some surfactant becomes turbid and separates into two isotropic phases under varied conditions of temperature 
and pressure or when appropriate substance is added to solution. The surfactant solution becomes turbid because it attains the cloud point temperature. At this point, the original surfactant solution separates into a surfactant phase of small volume, which is rich in the surfactant and containing the analyte or metal (organic or inorganic species) trapped by micellar structures and a bulk diluted aqueous phase $[57,58]$. The main advantage of cloud point technique is it uses an aqueous solution of the surfactants that are inexpensively resulting in lesser cost of reagents. Surfactants are not toxic, not volatile, and not easily fl ammable, unlike organic solvents. It is a simple, inexpensive, and highly fiefient meth od for the formation of micelles.

\section{Micellar microparticles or colloidal microparticles}

Microparticle entrapped micelles is a delivery system in which the particles of the micellar range are ensemble for drug and gene delivery in specific parts of the body. Microparticles containing micelles have the potential for delivering micelle encapsulated hydrophobic drug on targeted site [59-63]. Micelles have been used as drug carriers for administration and exhibit many advantages including their ease of production, long circulation times and having surfaces that are readily modified with targeting ligands.

It was previously shown that various hydrophobic agents could be successfully incorporated into micelles [64-66]. The major goals in designing colloidal microparticles topical formulation are to improve topical dosage forms that deliver the API locally (at site of application) in an efficient and effective manner, to tailor drug deposition, disposition and permeation kinetics through formulation engineering (altered composition, drug loading, droplet size, etc.).

Micellar delivery systems that are smaller than $100 \mathrm{~nm}$ can be readily prepared. The micelles can easily penetrate the tissue for targeted drug delivery but they disintegrate rapidly leading to faster release. The microparticles formation of these micelles can be an effective approach to sustain the release of the drug and also provide solid structures for further incorporation into a dosage form [67].

\section{Advantages of colloidal microparticles}

1. Have potential of delivering drug to targeted site

2. Allow the encapsulation of the hydrophobic drug

3. Are easy to produce and are economic

4. Improve topical dosage forms that deliver the API locally (at site of application) in an efficient and effective manner

5. Tailor drug deposition, disposition and permeation kinetics

\section{Sustain the drug release}

The application of micellar drug delivery system is owing to the improvement of bioavailability, minimization of drug degradation, prevention of harmful side effects. Micellar systems can solubilize poorly soluble drugs and thus increase the bioavailability of those drugs. It is recognized that solubilization in aqueous surfactant solutions at surfactant concentrations exceeding the critical micelle concentration is the means to formulate a variety of slightly soluble to practically insoluble compounds in the solution state [68]. Micellar solubilization improves the solubility of drugs. Micelle entrapped into microparticle is a sensible approach of converting an aqueous micellar solution into solid for ease of administration and patient compliance.

\section{Literature reviewed on microparticles entrapped micelles}

It was showed that reverse micelles made of amphiphilic phosphazene polymers can enhance the encapsulation efficiency and the in vitro release profile of poly (lactide-co-glycolide) (PLGA) microparticles. While plain PLGA microparticles released more than $50 \%$ of the drug in $1 \mathrm{~d}$, optimal micelle formulations encapsulating PLGA particles released less than $30 \%$ of the drug at the end of $60 \mathrm{~d}$. Microparticles are enabling the use of micelles as sustained release delivery systems. In order to sustain drug delivery beyond $2 \mathrm{mo}$, for instance up to 6 mo or a few years, microparticles and implant delivery systems are currently suitable options [69].
A new platform was developed for the pulmonary delivery of the therapeutic photosensitizing drug, hematoporphyrin ( $\mathrm{Hp}$ ) in the form of microparticles containing micelles in which the drug was encapsulated. Micellar $\mathrm{Hp}$ was formulated using different poloxamers and one of these, Pluronic L122-Hp, was subsequently incorporated into lactose microparticles by spray-drying. Various Spectral and morphological analysis was carried out before and after the dissolution of the microparticles in the water on both micellar $\mathrm{Hp}$, and lactose microparticles containing micellar Hp (lactosemicellar Hp). The Photodynamic activity of the various Hp samples were evaluated in human lung epithelial carcinoma A549 cells using a light-emitting diode (LED) device at a wavelength of $630 \pm 5 \mathrm{~nm}$. It was noted that the spectral properties, as well as the photodynamic activity of the micellar $\mathrm{Hp}$, were similar to that of microparticles formulated by spray-drying. Microparticles containing micelles have the potential for delivering micelle-encapsulated hydrophobic drugs in targeted therapy of pulmonary diseases [70].

It was reported that MEM technology containing drug entrapped micelles can successfully preserve the properties and integrity. Valsartan (VAL) was solubilized in cremophor EL micelles at cloud point temperature; lactose was dissolved in micellar dispersion and the dispersion was directly spray-dried to obtain solid product, which was subsequently converted into tablets using suitable excipients. Improved dissolution performance of valsartan tablets was observed when VAL tablets were produced by applying MEM technology. These tablets exhibited superior dissolution rate over controls and marketed tablets in all media employed irrespective of $\mathrm{pH}$ conditions and composition [71].

The dissolution rate of naproxen using micellar solubilization technology was studied. Cloud point temperature of the non-ionic surfactants was used as the technique for the solubility study. Cloud point technique is helpful in solubilizing the maximum amount of drug into the surfactant at the cloud point temperature. Polysorbate 80 was used to solubilize naproxen at cloud point temperature to form micelles further lactose was dissolved in micellar dispersion and the dispersion was directly spray-dried to obtain microparticles. These microparticles were converted into tablets using suitable excipients. It was found that the dissolution rate was higher in all the dissolution media irrespective of the $\mathrm{pH}$ conditions in both spraydried naproxen particles and directly compressed tablets [72].

The formulation of fenofibrate modified by MEM technology (FBMEM) was prepared. Fenofibrate was solubilized through CPT in various surfactants such as Tween 60, 80, Kolliphor P407, Gelucire 44/14, Acrysol K140 at different concentrations of $0.5,1,3,5 \% \mathrm{v} / \mathrm{v}$. These micellar dispersions were then dried with the help of a spray dryer and solid particles were obtained. The fenofibrate microparticles were then filled in hard capsules and showed higher dissolution profile. The obtained MEM were further analysed for DSC and FTIR spectra. It was found that the use of Acrysol K140 as surfactant enhanced the solubility of fenofibrate up to $644 \mathrm{mg}$ fenofibrate/1g surfactant. No chemical interactions between fenofibrate and Acrysol K140 were observed by DSC diagram and FTIR spectra [73].

\section{CONCLUSION}

The delivery of hydrophobic drug to the targeting site is a challenging task for the researchers because of its limited solubility. Here, the focus is given for the hydrophobic drug that needs to be delivered topically. Micelles provide the rapid disintegration of the drug at the targeted site. Micellar microparticles improve the solubility and dissolution of the poorly soluble drugs and provide drug delivery to a targeted site whereby release of the drug is sustained. Such advantages of micellar microparticles provide the big scope in future for the delivery of hydrophobic drug topically with more efficacy and less production cost.

\section{ACKNOWLEDGMENT}

We wish to express our gratefulness to Dr. S. L. Harikumar (Dean USPS), Sr. Gurvinder Singh Bahra (Honorable Chancellor), Honorable Vice Chancellor of Rayat and Bahra University Mohali (Punjab) for their praiseworthy inspiration, platform and constant support for the completion of this study. 


\section{AUTHORS CONTRIBUTIONS}

All the author have contributed equally

\section{CONFLICT OF INTERESTS}

Declared none

\section{REFERENCES}

1. Atiyeh B. Wound cleansing, topical antiseptics and wound healing. Int Wound J 2009;1:420-30.

2. Sah SK, Badola A, Mukhopadhyay S. Development and evaluation of tioconazole loaded emulgel. Int J Appl Pharm 2017;9:83-90.

3. Chang RK, Raw A, Lionberger R, Yu L. Generic development of topical dermatologic products: formulation development, process development, and testing of topical dermatologic products. Am Assoc Pharm Sci J 2013;15:41-52.

4. Tadwee IK, Gore S, Giradkar SP. Advances in topical drug delivery system: a review. Int J Pharm Res Appl sci 2011;1:14-23.

5. Kumar KK, Sasikanth K, Sabareesh M, Dorababu N. Formulation and evaluation of diacerein cream. Asian J Pharm Clin Res 2011;4:93-8.

6. Shaikh AC, Quazi A, Nazim S, Majaz Q, Siraj S, Khan T, et al. Formulation optimization of hydrodynamically balanced oral controlled release bioadhesive tablets of tramadol hydrochloride. Asian J Pharm Clin Res 2011;4:61-70.

7. Sruthi TP, Noby T, Daisy PA, Carla B. Microsponge drug delivery system for topical delivery-a review. Int J Pharm Chem Biol Sci 2016;6:424-31.

8. Sah S, Badola A, Nayak B. Emulgel: magnifying the application of topical drug delivery. Ind J Pharm Biol Res 2017;5:25-33.

9. Kshirsagar N. Drug delivery systems. Indian J Pharmacol 2000;32:S54-S61.

10. Zi P, Yang X, Kuang H, Yang Y, Yu L. Effect of HPbeta CD on solubility and transdermal delivery of capsaicin through rat skin. Int J Pharm 2008;358:151-8.

11. Rathbone M, Hadgraft J, Roberts M, Lane M. Dermal and transdermal drug delivery. Modified-release drug delivery technology. 2nd ed. U. K. Informa healthcare; 2002. p. 263-71.

12. Date A, Naik B, Nagarsenker M. Novel drug delivery systems: potential in improving topical delivery of antiacne agents. Skin Pharmacol Physiol 2006;19:2-16.

13. Foldvari M. Non-invasive administration of drugs through the skin: challenges in delivery system design. Pharm Sci Technol Today 2000;3:417-25.

14. Elsayed M, Abdallah O, Naggar V, Khalafallah N. Lipid vesicles for skin delivery of drugs: reviewing three decades of research. Int J Pharm 2007;332:1-16.

15. Dhote V, Bhatnagar P, Mishra PK, Mahajan SC, Mishra DK. Iontophoresis: a potential emergence of a transdermal drug delivery system. Sci Pharm 2012;80:1-28.

16. Garg T, Rath G, Goyal AK. Comprehensive review on additives of topical dosage forms for drug delivery. Drug Delivery 2014;22:969-87.

17. Nair A, Jacob S, Al-Dhubiab B, Attimarad M, Harsha S. Basic considerations in the dermatokinetics of topical formulations. Braz J Pharm Sci 2013;49:423-34.

18. Zhang $Q$, Grice JE, Wang G, Roberts MS. Cutaneous metabolism in transdermal drug delivery. Curr Drug Metab 2009;10:227-35.

19. Fox LT, Gerber M, Du Plessis J, Hamman JH. Transdermal drug delivery enhancement by compounds of natural origin. Molecules 2011;16:10507-40.

20. Tadwee IK, Gore S, Giradkar SP. Advances in topical drug delivery system: a review. Int J Pharm Res Appl Sci 2011;1:14-23.

21. Kikwai L, Babu RJ, Prado R, Kolot A, Armstrong CA, Ansel JC, et al. In vitro and in vivo evaluation of topical formulations of spantide II. AAPS Pharm Sci Tech 2005;6:565-72.

22. Moshfeghi AA, Peyman GA. Micro-and nanoparticulates. Adv Drug Delivery Rev 2005;57:2047-52.

23. Rosen $\mathrm{H}, \mathrm{Abribat} \mathrm{T}$. The rise and rise of drug delivery. Nat Rev Drug Discovery 2005;4:381-5.

24. Zi P, Yang X, Kuang H, Yang Y, Yu L. Effect of HPbeta CD on solubility and transdermal delivery of capsaicin through rat skin. Int J Pharm 2008;358:151-8.
25. Rathbone M, Hadgraft J, Roberts M, Lane M. Dermal and transdermal drug delivery. Modified-release drug delivery technology. 2nd ed. U. K. Informa healthcare; 2002. p. 263-71.

26. Ajazuddin A, Alexander A, Khichariya S, Gupta RJ, Patel TK. Recent expansions in an emergent novel drug delivery technology: Emulgel. J Controlled Release 2013;171:122-32.

27. Yousef $\mathrm{H}$, Sharma S. Anatomy, skin (Integument), Epidermis. Treasure Island (FL): Stat Pearls Publishing; 2018.

28. Wickett RR, Visscher MO. Structure and function of the epidermal barrier. Am J Infect Controlled 2006;34:S98-S110.

29. Dev A, Chodankar R, Shelke O. Emulgels: a novel topical drug delivery system. Pharma Bio Eval 2015;2:64-75.

30. Panwar S, Upadhyay N, Bairagi M, Gujar S, Darwhekar GN, Jain DK. Emulgel: a review. Asian J Pharm Life Sci 2011;1:333-42.

31. Dadwal M. Emulgel: a novel approach to topical drug delivery. Int J Pharm Bio Sci 2013;4:847-56.

32. Kute SB, Saudagar RB. Emulsified gel a novel approach for delivery of hydrophobic drugs: an overview. J Adv Pharm Edu Res 2013;3:368-76.

33. Khullar R, Saini S, Seth N, Rana AC. Emulgels: a surrogate approach for topically used hydrophobic drugs. Int J Pharm Bio Sci 2011;1:117-28.

34. Waugh A, Grant A. Ross and Wilson: anatomy and pharmacology in health and illness. Churchill Living stone; 2004. p. 361-4.

35. Rieger MM, Lachman L, Lieberman HA, Kanig JL. The theory and practice of industrial pharmacy. 3rd ed. PA Lea and Febiger, Philadelphia; 1986. p. 502-33.

36. Ruela ALM, Perissinato AG, Lino MES, Mudrik PS, Pereira GR. Evaluation of skin absorption of drugs from topical and transdermal formulations. Braz J Pharm Sci 2016;52:527-44.

37. Singh MD, Mital N, Kaur G. Topical drug delivery systems: a patent review. Expert Opin Ther Pat 2016;26:213-28.

38. Lachman L, Lieberman HA, Kang JL. Theory and practice of industrial pharmacy. Bombay, India: Verghese Publishing House; 1991. p. 534-63.

39. Lu PJ, Weitz DA. Colloidal particles: crystals, glasses, and gels. Annu Rev Condens Matter Phys 2013;4:217-33.

40. Sharma J, Kalra S, Sharma A, Rani S. Colloidal drug carriers. J Fam Pract 2009;9:21-37.

41. Vyas A, Kumar SA, Gidwani B. Carrier-based drug delivery system for the treatment of acne. Sci World J 2014:1-14. http://dx.doi.org/10.1155/2014/276260

42. Kevin L, Helen B. A review of the formation and classification of amphiphilic block copolymer nanoparticulate structures: micelles, nanospheres, nanocapsules and polymersomes. Eur J Pharm Biopharm 2007;65:259-69.

43. Husseini GA, Pitt WG. Micelles and nanoparticles for ultrasonic drug and gene delivery. Adv Drug Delivery Rev 2008;60:1137 52.

44. Markus J, Per H, Katarina E. Spherical micelles and other selfassembled structures in dilute aqueous mixtures of poly (Ethylene Glycol) lipids. J Phys Chem B 2001;105:8420-30.

45. Shota F, Shimpei Y, Sakiko M, Genki K, Kenta Y, Eri T, et al. Platonic micelles: monodisperse micelles with discrete aggregation numbers corresponding to regular polyhedra. Sci Rep. 2017;7:44494.

46. Lombardo D, Calandra P, Barreca D, Magazù S, Kiselev M. Soft interaction in liposome nanocarriers for therapeutic drug delivery. J Nanomater 2016;6:125.

47. Pelizzetti E, Pramauro E. Analytical applications of organized molecular assemblies. Anal Chim Acta 1985;169:1-29.

48. Hofland HEJ, Bouwstra JA, Spies F, Bodde HE, Nagelkerke JF, Cullander C. Interactions between non-ionic surfactant vesicles and human stratum corneum in vitro. J Liposome Res 1995;5:241-63.

49. Abraham W, Downing DT. Interaction between corneocytes and stratum corneum lipid liposomes in vitro. Biochim Biophys Acta 1990;1021:119-25.

50. Zellmer S, Pfeil W, Lasch J. Interaction of phosphatidylcholine liposomes with the human stratum corneum. Biochim Biophys Acta 1995;1237:176-82.

51. Coderch L, Fonollosa J, De Pera M, Estelrich J, De La Maza A, Parra JL. Influence of cholesterol on liposome fluidity by EPR. 
Relationship with percutaneous absorption. J Controlled Release 2000;68:85-95.

52. Mezei M, Gulasekharam V. Liposomes-a selective drug delivery system for the topical route of administration. Lotion dosage form. Life Sci 1980;26:1473-7.

53. Mezei M, Gulasekharam V. Liposomes-a selective drug delivery system for the topical route of administration: gel dosage form. J Pharm Pharmacol 1982;34:473-4.

54. Foldvari M, Gesztes A, Mezei M. Dermal drug delivery by liposome encapsulation: clinical and electron microscopic studies. J Microencapsul 1990;7:479-89.

55. Bilgicer B. Biomedical Engineering; 2009.

56. Aliabadi H, Lavasaniafar A. Polymeric micelles for the drug delivery. Expert Opin Drug Delivery 2006;3:139-62.

57. Hinze WL, Pramauro E. A critical review of surfactant-mediated phase separations (cloud point extractions)-theory and applications. Crit Rev Anal Chem 1993;24:133-77.

58. Pereira MG, Arruda MAZ. Trends in pre-concentration procedures for metal determination using atomic spectrometry techniques. Microchim Acta 2003;141:115-31.

59. Opanasopit P, Yokoyama M, Watanabe M, Kawano K, Maitani Y, Okano T. Block copolymer design for camptothecin incorporation into polymeric micelles for passive tumor targeting. Pharm Res 2004;21:2001-8.

60. Cai S, Vijayan K, Cheng D, Lima EM, Discher DE. Micelles of different morphologies-advantages of worm-like filomicelles of PEO-PCL in paclitaxel delivery. Pharm Res 2007;24:2099-109.

61. Hofman JW, Carstens MG, Van Zeeland F, Helwig C, Flesch FM, Hennink WE. Photocytotoxicity of mTHPC (temoporfin) loaded polymeric micelles mediated by lipase catalyzed degradation. Pharm Res 2008;25:2065-73.

62. Vakil R, Knilans K, Andes D, Kwon GS. Combination antifungal therapy involving amphotericin $B$, rapamycin and 5fluorocytosine using PEG-phospholipid micelles. Pharm Res 2008;25:2056-64.
63. Yang Y, Chen C, Yang J, Tsai T. Spray-dried microparticles containing polymeric micelles encapsulating hematoporphyrin. AAPS J 2010;12:138-46.

64. Jette KK, Law D, Schmitt EA, Kwon GS. Preparation and drug loading of poly (ethylene glycol)-block-poly (Ecaprolactone) micelles through the evaporation of a cosolvent azeotrope. Pharm Res 2004;21:1184-91.

65. Djordjevic J, Barch M, Uhrich KE. Polymeric micelles based on amphiphilic scorpion-like macromolecules: novel carriers for water-insoluble drugs. Pharm Res 2005;22:24-32.

66. Mourya VK, Inamdar N, Nawale RB, Kulthe SS. Polymeric micelles: general considerations and their applications. Ind J Pharm Edu Res 2011;45:128-38.

67. Fournier E, Dufresne MH, Smith DC, Ranger M, Leroux JC. A novel one-step drug-loading procedure for water soluble amphiphilic nanocarriers. Pharm Res 2004;21:962-8.

68. Liu B, Yang M, Li R, Ding Y, Qian X, Yu L, Jiang X. The antitumor effect of novel docetaxel-loaded thermosensitive micelles. Eur J Pharm Biopharm 2008;69:527-34.

69. Qiu LY, Zhang JX, Yan MQ, Jin Y, Zhu KJ. Reverse self-assemblies based on amphiphilic polyphosphazenes for encapsulation of water-soluble molecules. Nanotech 2007;18:475-602.

70. Yang Y, Chen C, Yang J, Tsai T. Spray-dried microparticles containing polymeric micelles encapsulating hematoporphyrin. AAPS J 2010;12:138-46.

71. Maheswari PD, Rambhau D, Narasu ML. Microparticlesentrapped micelles: a novel delivery system to improve solubility and dissolution rate of poorly water-soluble valsartan. J Microencapsul 2013;30:805-16.

72. Maheswari PD, Rambhau D, Narasu ML. Micellar solubilization in the formulation development of poorly soluble naproxen. Pharm Reg Affairs 2013;2:108.

73. Tran QT, Vu THM, Tran VT. Preparation of $200 \mathrm{mg}$ fenofibrate hard capsule with high dissolution profile with microparticle entrapped micelles technology. J Pharm Sci 2017;44:50-7. 